\title{
Impact of Radial and Angular Sampling on Multiple Shells Acquisition in Diffusion MRI
}

\author{
Sylvain Merlet ${ }^{1}$, Emmanuel Caruyer $^{1}$, and Rachid Deriche ${ }^{1}$ \\ Athena Project-Team, INRIA Sophia Antipolis - Méditerranée, France
}

\begin{abstract}
We evaluate the impact of radial and angular sampling on multiple shells (MS) acquisition in diffusion MRI. The validation of our results is based on a new and efficient method to accurately reconstruct the Ensemble Average Propagator (EAP) in term of the Spherical Polar Fourier (SPF) basis from very few diffusion weighted magnetic resonance images (DW-MRI). This approach nicely exploits the duality between SPF and a closely related basis in which one can respectively represent the EAP and the diffusion signal using the same coefficients. We efficiently combine this relation to the recent acquisition and reconstruction technique called Compressed Sensing (CS). Based on results of multi-tensors models reconstruction, we show how to construct a robust acquisition scheme for both neural fibre orientation detection and attenuation signal/EAP reconstruction.
\end{abstract}

Keywords: Diffusion MRI, Compressed sensing, Ensemble Average Propagator recovery, Propagator, Orientation Distribution Function, Spherical Polar Fourier, Multiple Shells Sampling.

\section{Introduction}

Since the introduction of CS by [5], this method has been used in a large range of domains including image and video compression as well as geophysics and medical imaging. In 8, we apply CS in diffusion magnetic resonance imaging (dMRI) for modelling the EAP, in SPF basis introduced by [1, from highly under sampled diffusion weighted MR images (DW-MRIs).

In [8, the quality of reconstruction is sensitive to the acquisition scheme. Hence, in order to remove the variance of the results due to the random aspect of the sampling scheme, it is necessary to find a robust and efficient way to acquire DW-MRIs. For this purpose, we propose to evaluate and compare several sampling protocols. In this study, we begin in 2 by summarizing the EAP based CS-reconstruction proposed in [8]. The section 3 aims to describe some techniques to build sampling scheme. Finally in section 4 we present several sets of experiments in order to examine the robustness and efficiency of these schemes.

\section{EAP Based CS Reconstruction}

The method described in [8] enables the modeling of the EAP in the SPF basis, using the recent technique known as Compressed Sensing (CS). The method 
allows us to analytically reconstruct the propagator at any radius and, also, to derive one of its famous feature: the Orientation Distribution Function (ODF). The CS reconstruction is based on a $l_{1}$ minimization scheme promoting the signal sparsity. For a more mathematical definition, suppose our signal of interest is a vector $\mathbf{x} \in \mathbb{R}^{m}$. Let $\mathbf{y} \in \mathbb{R}^{n}$, with $n \ll m$, be an observation representative of $\mathbf{x}$ given by the sensor of a given application. $\mathbf{y}=\mathbf{A x}+\eta$, where $\mathbf{A} \in \mathbb{R}^{n \times m}$ is the measurement matrix, so called the CS matrix, and $\eta \in \mathbb{R}^{n}$ represents the acquisition noise. Our goal is to find $\mathbf{x}$ given the measurement vector $\mathbf{y}$. Since $\mathbf{y}$ has less entries than $\mathbf{x}$, this ill-posed problem cannot be resolved without any prior knowledge about the signal to recover.

We condider the signal admits a sparse representation with respect to an orthonormal basis $B$. $\mathbf{c} \in \mathbb{R}^{n_{c}}$ is the vector of transform coefficients $\left\{c_{i}=\right.$ $\left.\left\langle x, b_{i}\right\rangle, b_{i} \in B, i=1, \ldots, n_{c}\right\}$. We can constrain most of the transform coefficients to be zero by minimizing the $l_{1}$ norm defined by $\|\mathbf{c}\|_{1}=\sum_{i=1}^{n_{c}}\left|c_{i}\right|$ [7]. The solution $\mathbf{x}$ of our problem is given by solving the following convex optimization problem :

$$
\arg \min _{\mathbf{x}} J(\mathbf{x})=\|\mathbf{A x}-\mathbf{y}\|_{2}^{2}+\lambda\|\mathbf{c}\|_{1}
$$

The first term is the data consistency constraint, $\|\mathbf{c}\|_{1}$ is the sparsity constraint. $\lambda$ is the Lagrange parameter that balances the confidence between the measured signal $\mathbf{y}$ and the sparsity constraint. The data consistency constraint enables the solution to remain close to the raw data acquisition, whereas the minimization of the second term promotes sparsity. In short, this mathematical problem searches for the sparsest solution while remaining close to the acquired data.

In our previous paper [8], we define $B$ as the so called Spherical Polar Fourier (SPF) basis in spherical coordinates $(r, \theta, \varphi)$. This orthonormal basis is the combination of the real spherical harmonics $Y_{m}^{l}$ and the Gauss-Laguerre functions $R_{n}$. It is expressed as $\Psi_{n \ell m}(r, \theta, \phi)=R_{n}(r) Y_{m}^{\ell}(\theta, \phi)$, where $\ell \in \mathcal{N}$ is the spherical harmonic order, $-l \leq m \leq l$ the $\mathrm{SH}$ degree and $n$ the Gauss-Laguerre order. This basis enables a complete description of the diffusion propagator.

We described a method to accurately reconstruct the EAP $P$ from undersampled measurements. In this method $P$ was estimated by a truncated linear combination of the SPF basis functions $\Psi_{n \ell m}$

$$
P(r, \theta, \phi)=\sum_{n=0}^{N} \sum_{\ell=0}^{L} \sum_{m=-\ell}^{\ell} c_{n \ell m} \Psi_{n \ell m}(r, \theta, \phi),
$$

where $c_{n \ell m}=\left\langle P, \Psi_{n \ell m}\right\rangle$ are the SPF transform coefficients.

While modelling the EAP with respect to the SPF basis, we have shown that we can reconstruct the corresponding attenuation signal $E$ by keeping the same coefficients $\left\{c_{n \ell m}, n=0, \ldots, N, l=0, \ldots, L, m=-l, \ldots, l\right\}$ but by using a new family of functions called the SPF dual basis $\left\{\Phi_{n \ell m}, n=0, \ldots, N, l=\right.$ $0, \ldots, L, m=-l, \ldots, l\} . E$ can, thus, be written as

$$
E(q, \theta, \varphi)=\sum_{n=0}^{N} \sum_{\ell=0}^{L} \sum_{m=-\ell}^{\ell} c_{n \ell m} \Phi_{n \ell m}(q, \theta, \phi)
$$


where $q=|\mathbf{q}|$ is the norm of the effective gradient $\mathbf{q}$ in q-space and $\theta, \varphi$ the direction angles. Suppose $n_{q}$ is the number of measurement samples, $\mathbf{E} \in \mathbb{R}^{n_{q}}$ a vector representing the signal attenuation, $\mathbf{c} \in \mathbb{R}^{n_{c}}$ a vector of the SPFd coefficients $c_{n \ell m}$ and $\boldsymbol{\Phi} \in \mathbb{R}^{n_{q} \times n_{c}}$ the matrix constructed with the SPFd basis functions

$$
\mathbf{\Phi}=\left(\begin{array}{ccc}
\Psi_{n \ell m}\left(r_{1}, \theta_{1}, \phi_{1}\right) & \cdots & \Psi_{N L L}\left(r_{1}, \theta_{1}, \phi_{1}\right) \\
\vdots & \ddots & \vdots \\
\Psi_{n \ell m}\left(r_{n_{q}}, \theta_{n_{q}}, \phi_{n_{q}}\right) & \cdots & \Psi_{N L L}\left(r_{n_{q}}, \theta_{n_{q}}, \phi_{n_{q}}\right)
\end{array}\right)
$$

We can write equation (3) as an over determined linear system, $\mathbf{E}=\mathbf{\Phi c}$ Let $\boldsymbol{\Phi}_{u} \in \mathbb{R}^{n_{u} \times n_{c}}$ be the undersampled version of $\boldsymbol{\Phi}$ operator and $\mathbf{E}_{u} \in \mathbb{R}^{n_{u}}$ the vector of undersampled signal attenuation. We can rewrite the problem described in equation 1

$$
\arg \min _{\mathbf{c}} J(\mathbf{c})=\left\|\mathbf{\Phi}_{\mathbf{u}} \mathbf{c}-\mathbf{E}_{\mathbf{u}}\right\|_{2}^{2}+\lambda\|\mathbf{c}\|_{1}
$$

Eq. (5) searches for the EAP coefficients with respect to the SPF basis, i.e. we can compute a continuous version of the true propagator. Using the same coefficients, we can as well model the attenuation signal with respect to the SPFd basis functions. Moreover, in 8] we give an analytical estimate of the ODF in terms of spherical harmonic functions and the coefficients $c_{n \ell m}$.

In [8], we randomly took 80 measurements spread on 3 shells with b values $1000,2000,3000 \mathrm{~s} / \mathrm{mm}^{2}$. However, the random aspect of the acquisition process makes the reconstruction very sensitive to the sampling scheme. We selected it as follow: on 100 sampling schemes generated, we kept the one that leads to the best results. In this way, good results were observed while performing the reconstruction on our data set but were not observed in all cases. In the next section, we propose several sampling schemes in order to make the method more robust to this reconstruction phase.

\section{Sampling Design}

\subsection{Jones}

References 64 give an algorithm to uniformly distribute $\mathrm{N}$ points $q_{n} \in R^{3}$ on a sphere by considering each point as an antipodal pair of electrical charges. The method involves the minimization of the electrostatic force of repulsion between each couple of charges. The electrostatic repulsion between two points $q_{i}$ and $q_{j}$ is given by

$$
E\left(q_{i}, q_{j}\right)=\frac{1}{\left\|q_{i}+q_{j}\right\|}+\frac{1}{\left\|q_{i}-q_{j}\right\|}
$$


For a set of $\mathrm{N}$ points, the energy to minimize becomes

$$
J_{J}=\sum_{i \neq j} E\left(q_{i}, q_{j}\right)
$$

Reference [3] provides Camino, an Open-Source Diffusion-MRI Reconstruction and Processing software. They include several sets of directions, from $\mathrm{N}=3$ to 150 points, computed by electrostatic energy minimization.

\subsection{Generalized Jones}

This method is proposed by [2] as a generalization of [6] to multi-shells acquisition. It enables the distribution of $\mathrm{N}$ points $q_{n} \in R^{3}$ on $\mathrm{K}$ shells of radius $r_{k}$. The points from each shell have staggered directions and follow a near-optimal uniform distribution. Another important point in this method is the possibility to balance the proportion $\alpha_{k}$ of samples between shells. We will take advantage of this feature in order to test out different spherical distributions.

Firstly, the method consists in minimizing the electrostatic repulsion between every point for each shell independently, that is

$$
E_{1}=\sum_{k} r_{k} \alpha_{k} \sum_{i \neq j \text { s.t }\left\|q_{i}\right\|=\left\|q_{j}\right\|=r_{k}} E\left(q_{i}, q_{j}\right)
$$

Then, in order to have staggered directions between shells, 2 introduces a new term that minimizes the electrostatic repulsion of the $\mathrm{N}$ points projected on the unit sphere. It comes to minimize

$$
E_{2}=\sum_{i \neq j} \frac{1}{\left\|\frac{q_{i}}{\left\|q_{i}\right\|}-\frac{q_{j}}{\left\|q_{j}\right\|}\right\|}+\frac{1}{\left\|\frac{q_{i}}{\left\|q_{i}\right\|}+\frac{q_{j}}{\left\|q_{j}\right\|}\right\|}
$$

Finally, the energy to minimize is $J_{G J}=(1-\mu) E_{1}+\mu E_{2}$, where $\mu$ is a weighting factor.

\subsection{Sampling Schemes}

We perform our experiments on five sampling schemes to evaluate the impact of the angular sampling in MS acquisition : Regular sampling (RS) means we take the same directions on each shell. These directions are provided by the application of Jones algorithm. Uniform Jones sampling (UJS) uses the generalized Jones algorithm by setting the parameters in such way that the samples are distributed along a spherical uniform law (The number of point on each shell is proportional to the square of its radius). Constant Jones sampling (CJS) uses the generalized Jones algorithm by setting the parameters in such way that there is a constant number of samples by shell. Constant random sampling (CRS) means we randomly take directions on each shell by setting a constant number of samples by shell. Uniform random sampling (URS) means we randomly take directions on each shell in such way that the samples are distributed along a spherical uniform law. 


\section{Experimental Results}

In this section, we review the outcome of angular sampling as well as radial sampling on the CS reconstruction defined in section 2. The performance of each sampling scheme is determined on both attenuation signal reconstruction and neural fibre orientation which is given by the maxima of the estimated ODF estimated as in 8 . We evaluate the maxima extraction by the Percentage of Corrected Number of Detected Maxima (PCNDM) obtained on a predefined number of trial. Each time the number of detected maxima $N_{m}$ is correct we also compute the Angular Error in degrees $\frac{1}{N_{m}} \sum_{m=0}^{N_{m}} \frac{180}{\pi} \arccos \left(\tilde{u}_{m} \cdot u_{m}\right)$, where $\tilde{u}_{m}$ is the orientation of the detected maxima and $u_{m}$ the ground truth. The mean over all the trial gives the Mean Angular Error (MAE).

The quality of the signal attenuation estimation $\tilde{S}$ is given by the Normalized Mean Square Error (NMSE). For N sampling points $q_{n}$ the NMSE is $\frac{\sum_{i=1}^{N}\left|S\left(q_{i}\right)-\tilde{S}\left(q_{i}\right)\right|^{2}}{\sum_{i=1}^{N}\left|S\left(q_{i}\right)\right|^{2}}$, where $S$ is the ground truth signal attenuation. Then, we average the NMSE obtained in all the trials.

Before initiating the procedure, we set some reconstruction parameters. SNR $\zeta=20$, for average quality data. In eq. [5] we set $\lambda$ to 0.01 as in [8].

We reconstruct the propagator from a multi-Gaussian model through four scenarios : One fiber, two $60^{\circ}$-crossing fibres, two $70^{\circ}$-crossing fibres, two $90^{\circ}$ crossing fibres. All the results are obtained on 1500 independant trials.

Angular Profile: For each scenario we use three shells with b values equal to 500,1500 , and 3000 . We begin by evaluate the five sampling schemes presented in section 3.3 while using only $\mathrm{N}=60$ samples in the reconstruction. Figure 1 presents the results through the computation of the Percentage of Corrected Number of Detected Maxima (PCNDM), the Mean of Angular Error (MAE) in degrees and the Normalized Mean Square Error (NMSE) respectively on line 1, 2 and 3. Each color corresponds to one the five sampling schemes. Each group of five bars correspond to one of the scenarios presented at the beginning of the section (One fiber, two $60^{\circ}$-crossing fibres, two $70^{\circ}$-crossing fibres, two $90^{\circ}$ crossing fibres). We can see that, in term of PCNDM, two schemes stand out: the UJS and RS schemes (green and dark blue). However, due to the bad capacity of the RS scheme to resolve $60^{\circ}$-crossing fibres and most probably low degrees as well, we cannot rely upon it for maxima extraction. This is verified by looking at the MAE (second line) of RS scheme. Once again UJS gives the best results, i.e. the lowest MAE. Overall, orientation detection is better performed when a spherical uniform distribution is applied compared to a constant distribution. Constant distribution means we take the same number of samples on each shell. Random angular sampling confirms this point. Concerning the NMSE (third line), three sampling schemes are quite equivalents: the RS, the UJS and CJS schemes. Even if the regular one is slightly better than the other, it is difficult to distinguish one scheme from another. Here again random sampling does not meet the CS expectations.

We illustrate, as well, the evolution of the PCNDM, MAE and NMSE (from left to right in fig. 22) against the number of samples taken in the reconstruction. 


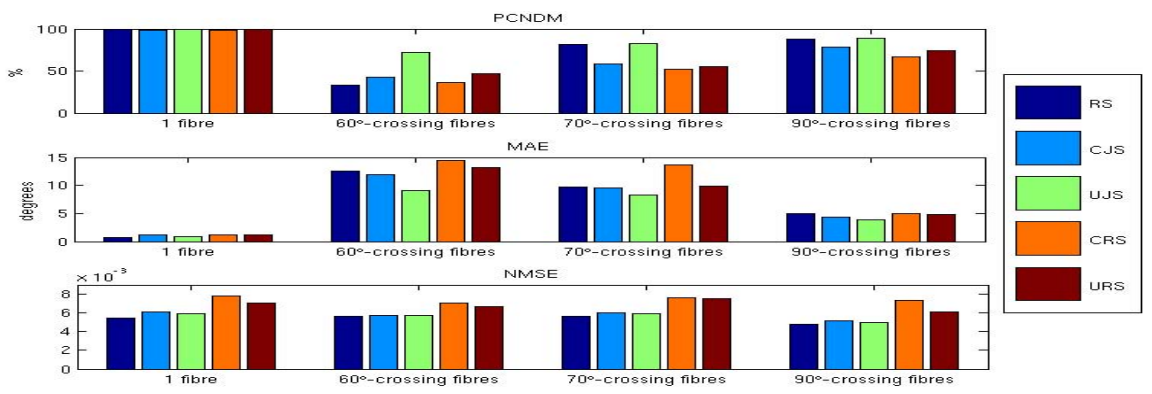

Fig. 1. Reconstruction results while using only $N=60$ samples in the reconstruction. Five angular sampling schemes are examined : Regular sampling (dark blue bars), Constant Jones sampling (light blue bars), Uniform Jones sampling (green bars) Constant random sampling (orange bars), Uniform random sampling (red bars). From top to bottom the three lines respectively represent the Percentage of Corrected Number of Detected Maxima (PCNDM), the Mean of Angular Error (MAE) in degrees and the Normalized Mean Square Error (NMSE). Each group of five bars correspond to one of the following scenarios : One fiber, two $60^{\circ}$-crossing fibres, two $70^{\circ}$-crossing fibres, two $90^{\circ}$-crossing fibres (from left to right).

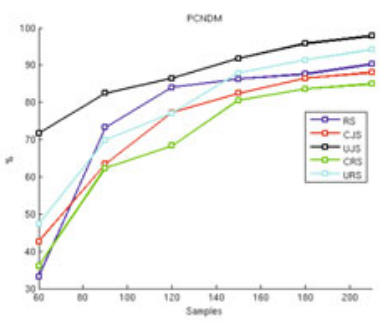

(a)

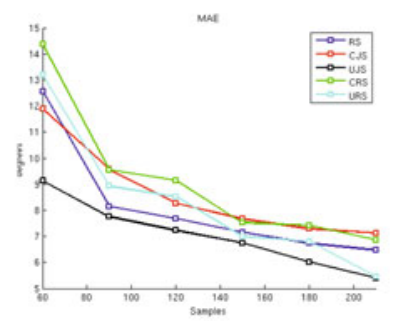

(b)

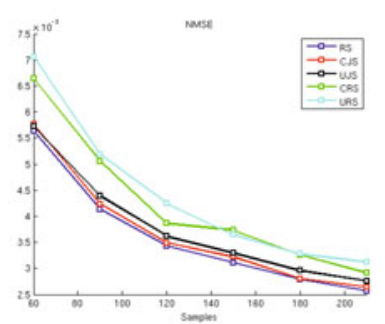

(c)

Fig. 2. Evolution of the reconstruction results against the number of samples for the $60^{\circ}$-crossing fibres. Five angular sampling schemes are examined : Regular sampling (dark blue), Constant Jones sampling (red), Uniform Jones sampling (black) Constant random sampling (green bars), Uniform random sampling (light blue). (a) represents the Percentage of Corrected Number of Detected Maxima (PCNDM), (b) the Mean of Angular Error (MAE) in degrees and (c) the Normalized Mean Square Error (NMSE).

Only the results for $60^{\circ}$-crossing fibres are represented. For maxima extraction (see PCNDM (a) and MAE (b)), the UJS overcomes the other schemes for every number of samples. In term of NMSE, the figure 2. c shows that the gap between the RS, the UJS and CJS is not sufficiently large in order to distinguish one scheme from another. Random sampling gives less stable curves than the others. It shows the problem of sensibility for this kind of scheme in our reconstruction. 
In conclusion with respect to the angular sampling, UJS is a robust and efficient way to build MS schemes.

Radial Profile: Now we want to examine the influence of the radial sampling. Due to the robustness of UJS, we decide to keep it while changing the number of shells from 1 to 12 .

Let us examine the figure 3(a) and (b). These results show that, above 3 shells, the MAE and the PCNDM do not vary a lot. It means the uniform sampling scheme is not sensitive to the number of shells used for the acquisition when fibre orientation detection is required. With 1 and 2 shells, we get a higher PCNDM than previously. However this improvement is done to the price of an increasing MAE. Moreover, when few shells are taken for sampling, it is more difficult to catch significant information of the radial profile. The $b$ values have to be cautiously chosen if few shells are used. By regularly taking more and more shells in a MS process, we cover more precisely the radial profile of our signal and we get rid of the choice of b-values.

Concerning the NMSE, figure 3(c) shows that increasing the number of sample decreases the NMSE until the number of shells exceeds a specific value. This limit may due to the fact that a too big increase of the radial resolution lead to a fall of the angular resolution. Figure 3(c) shows a limit of 9 shells in our case. However, we dont need to reach this point. Indeed the quasi-flatness of the curves before 9 shells allows us to use less shells for sampling while keeping the advantage of MS sampling.

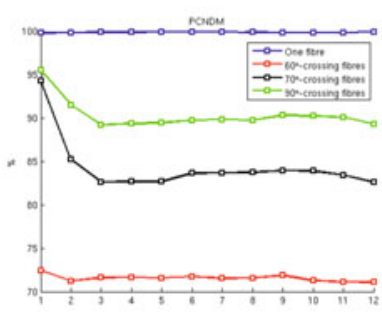

(a)

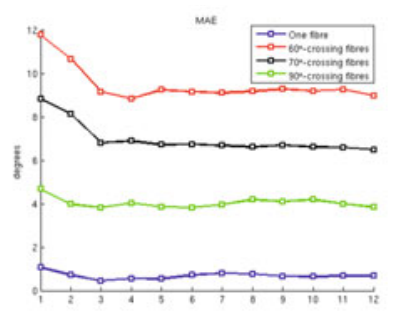

(b)

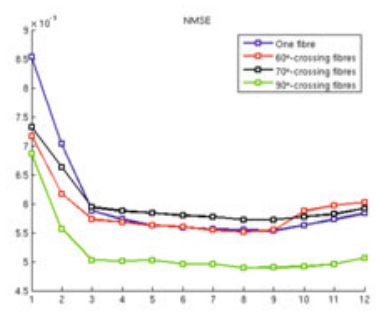

(c)

Fig. 3. Evolution of the reconstruction results against the number of shells. Uniform Jones sampling is used while changing the number of shells from 1 to 12. Four scenarios are examined : One fiber (blue curve), two $60^{\circ}$-crossing fibres (red curve), two $70^{\circ}$ crossing fibres (black curve), two $90^{\circ}$-crossing fibres (green curve). (a) represents the Percentage of Corrected Number of Detected Maxima (PCNDM), (b) the Mean of Angular Error (MAE) in degrees and (c) the Normalized Mean Square Error (NMSE).

Conclusion of the Experiments: With respect to the angular profile, one sampling scheme stand out : the UJS scheme. It allows us to correctly detect the orientation of neural fibres and especially for low number of samples $(\mathrm{N}=60)$. It also gives good results in terms of NMSE. The results show again that the 
signal attenuation reconstruction and maxima extraction are very sensitive to the sampling scheme. Even if random sampling (CRS, URS) can work better than near-regular sampling (RS, CJS, UJS), we cannot ensure that it will work in every case. Fibre orientation detection is not sensitive while changing the number of shells of the UJS scheme. A great advantage of MS sampling, compare to one shell sampling, lies in the quasi non-sensibility in the choice of b-values. Indeed we just need to acquire our signal on shells regularly spaced. On the other hand, due to the small number of point used, a compromise has to be done between radial and angular resolution.

\section{Conclusion}

The main contribution of this paper is to evaluate different way to sample q-space in dMRI. We showed that multiple shells acquisition is of great interest when dealing with fibres orientation detection and attenuation signal reconstruction. Hence this method generalizes the Q-ball imaging while being able to reconstruct the EAP and signal attenuation at any radius. In our study, we also showed that generalized Jones algorithm is a good way to build robust multiple shells sampling schemes and that the use of a spherical uniform distribution improves the results.

\section{References}

1. Assemlal, H.-E., Tschumperlé, D., Brun, L.: Efficient computation of PDF-based characteristics from diffusion MR signal. In: Metaxas, D., Axel, L., Fichtinger, G., Székely, G. (eds.) MICCAI 2008, Part II. LNCS, vol. 5242, pp. 70-78. Springer, Heidelberg (2008)

2. Caruyer, E., Lenglet, C., Sapiro, G., Deriche, R.: Incremental gradient table for multiple q-shells diffusion mri. In: HBM 17th Annual Meeting (June 2011)

3. Cook, P.A., Bai, Y., Nedjati-Gilani, S., Seunarine, K.K., Hall, M.G., Parker, G.J., Alexander, D.C.: Camino: Open-source diffusion-mri reconstruction and processing. In: 14th ISMRM, Seattle, USA (2006)

4. Deriche, R., Calder, J., Descoteaux, M.: Optimal real-time q-ball imaging using regularized kalman filtering with incremental orientation sets. Medical Image Analysis 13(4), 564-579 (2009)

5. Donoho, D.L.: Compressed sensing. IEEE Trans. on Information Theory 52(4), 1 (2004)

6. Jones, D., Horsfield, M., Simmons, A.: Optimal strategies for measuring diffusion in anisotropic systems by magnetic resonance imaging. Magnetic Resonance in Medicine 42(39), 515-525 (1999)

7. Lustig, M., Donoho, D., Pauly, J.: Sparse mri: The application of compressed sensing for rapid mr imaging. Magnetic Resonance in Medicine 58(6), 1182-1195 (2007)

8. Merlet, S., Cheng, J., Ghosh, A., Deriche, R.: Spherical polar fourier eap and odf reconstruction via compressed sensing in diffusion mri. In: Proceedings of ISBI (2011) 